\title{
The extended ROSAT-ESO Flux-Limited X-ray Galaxy Cluster Survey (REFLEX II)
}

\section{The mass function of galaxy clusters}

\author{
Hans Böhringer ${ }^{1}$, Gayoung Chon $^{1}$, and Masataka Fukugita ${ }^{2}$ \\ 1 Max-Planck-Institut für extraterrestrische Physik, 85748 Garching, Germany \\ e-mail: hxb@mpe.mpg.de \\ 2 Kavli Institute for the Physics and Mathematics of the Universe, University of Tokyo, 2778583 Kashiwa, Japan \\ Received 19 May 2017 / Accepted 16 August 2017
}

\begin{abstract}
The mass function of galaxy clusters is a sensitive tracer of the gravitational evolution of the cosmic large-scale structure and serves as an important census of the fraction of matter bound in large structures. We obtain the mass function by fitting the observed cluster X-ray luminosity distribution from the REFLEX galaxy cluster survey to models of cosmological structure formation. We marginalise over uncertainties in the cosmological parameters as well as those of the relevant galaxy cluster scaling relations. The mass function is determined with an uncertainty of less than $10 \%$ in the mass range $3 \times 10^{12}$ to $5 \times 10^{14} M_{\odot}$. For the cumulative mass function we find a slope at the low-mass end consistent with a value of -1 , while the mass-rich end cut-off is milder than a Schechter function with an exponential term $\exp \left(-M^{\delta}\right)$ with $\delta$ smaller than 1 . Changing the Hubble parameter in the range $H_{0}=67-73 \mathrm{~km} \mathrm{~s}^{-1} \mathrm{Mpc}^{-1}$ or allowing the total neutrino mass to have a value in the range $0-0.4 \mathrm{eV}$ causes variations less than the uncertainties. We estimate the fraction of mass locked up in galaxy clusters: about $4.4 \%$ of the matter in the Universe is bound in clusters (inside $r_{200}$ ) with a mass larger than $10^{14} M_{\odot}$ and $14 \%$ to clusters and groups with a mass larger than $10^{13} M_{\odot}$ at the present Universe. We also discuss the evolution of the galaxy cluster population with redshift. Our results imply that there is hardly any clusters with a mass $\geq 10^{15} M_{\odot}$ above a redshift of $z=1$.
\end{abstract}

Key words. galaxies: clusters: general - large-scale structure of Universe - X-rays: galaxies: clusters

\section{Introduction}

Clusters of galaxies form from peaks in the density fluctuation field in the Universe in a well prescribed way. The statistics of the peaks is clearly characterised for a given cosmological model. Therefore in the framework of the currently wellestablished cosmic structure formation theory, the abundance of galaxy clusters as a function of their mass can be predicted (e.g. Press \& Schechter 1974; Bardeen et al. 1986; Bond et al. 1991; Jenkins et al. 2001; Warren et al. 2006; Tinker et al. 2008; Despali et al. 2016). This cluster mass function is a very sensitive tracer of the growth of structure in the Universe and its observational determination can be applied to stringent tests of cosmological models. In most cases, in these cosmological tests the mass function is substituted by a function of another more easily observed cluster property which can be used as a good mass proxy, such as cluster richness, X-ray luminosity or temperature, or the Sunyaev-Zel'dovic effect decrement (e.g. Perenod 1980; Reiprich \& Böhringer 2002; Henry 2004; Vikhlinin et al. 2009; Rozo et al. 2010; Mantz et al. 2010; Böhringer et al. 2011; Planck Collaboration XX 2014; Planck Collaboration XXIV 2016; Böhringer et al. 2014; Böhringer \& Chon 2015).

Since it is very useful to know the cluster mass function for many applications, apart from the testing of cosmological models, as for example for models of the halo occupation distribution (e.g. Peacock \& Smith 2000; Berlind \& Weinberg 2002; Mehrtens et al. 2016), it is important to compile our knowledge on the mass function explicitly. For this purpose we use our X-ray cluster survey based on the ROSAT All-Sky Survey, which is currently the largest, statistically most complete and well described cluster survey in the nearby Universe covering most of the sky outside the galactic band. We focus here on the REFLEX II cluster survey in the southern sky, because in the northern counterpart, the NORAS II survey, there are still some cluster candidates with missing redshifts (Böhringer et al. 2017).

One conventional way to derive the mass function from observations is to use the $\mathrm{X}$-ray luminosity distribution and convert the data via an empirical X-ray luminosity-mass scaling relation. We like to explore a different approach. Since the theory of structure formation is well established and has been tested against many observational results, we have good theoretical background knowledge of the mass function and its relation to the cosmological model. We can use this as additional information to constrain the mass function by requesting that it is not only consistent with the data but also with structure-formation theory. In practice, this type of mass function appears as a byproduct when we test cosmological models, and does not require additional calculations. The method is described in detail in Sect. 2. It provides very tight constraints on the mass function.

The paper is structured as follows. Section 2 provides a brief account of the REFLEX cluster survey and its use for cosmological tests. Section 3 describes the method for deriving the mass function. In Sect. 4 we present the results and compare with previous observations. We look at further implications of our results in Sect. 5 including the fraction of cosmic 
matter bound in clusters, the implied redshift evolution of the mass function, and the predicted total number counts of clusters. Section 6 then provides a summary and conclusions. We quote and display all our results scaled by a Hubble parameter of $h_{70}=H_{0} / 70 \mathrm{~km} \mathrm{~s}^{-1} \mathrm{Mpc}^{-1}$.

\section{The REFLEX II galaxy cluster survey}

The REFLEX II galaxy cluster survey produced a catalogue of $911 \mathrm{X}$-ray luminous galaxy clusters down to a flux limit of $1.8 \times 10^{-12} \mathrm{erg} \mathrm{s}^{-1} \mathrm{~cm}^{-2}$ in the $0.1-2.4 \mathrm{keV}$ energy band with an estimated completeness of about $95 \%$ and a possible significant $\mathrm{X}$-ray contamination by active galactic nuclei (AGN) in about $6 \%$ of the sources (Böhringer et al. 2013). It is based on the X-ray detection of the clusters in the ROSAT All-Sky Survey (Trümper 1993; Voges et al. 1999) and covers a region in the southern sky below equatorial latitude $+2.5^{\circ}$ and at galactic latitude $\left|b_{\mathrm{II}}\right| \geq 20^{\circ}$. The regions of the Magellanic clouds have been excised. The total survey area is $\sim 4.24$ ster. The source detection, the galaxy cluster sample definition and compilation, and the construction of the survey selection function as well as tests of the completeness of the survey are described in Böhringer et al. (2013).

The X-ray fluxes and derived luminosities have been determined by means of the growth curve analysis method (Böhringer et al. 2000) within a fiducial cluster radius of $r_{500}{ }^{1}$. The radius $r_{500}$ is derived from the mass of the clusters, which is obtained by the X-ray luminosity-mass relation (Böhringer et al. 2014, Eq. (10)) obtained by Pratt et al. (2009), also consistent with that of Vikhlinin et al. (2009) $)^{2}$. The uncertainty of the flux and luminosity measurement is on average about $20 \%$.

In Böhringer et al. $(2014,2016)$ and Böhringer \& Chon (2015) we used the REFLEX cluster sample to test cosmological models by comparing the observed distribution of cluster X-ray luminosities in the cluster catalogue from the REFLEX II project with model predictions. The comparison is performed by means of a likelihood method described in detail in Böhringer et al. (2014). In these calculations the model for structure formation is based on a primordial power spectrum of the matter density fluctuations with a slope of 0.96 and a transfer function which models the evolution of the power spectrum obtained with the program CAMB (Lewis et al. 2000) ${ }^{3}$. The prediction of the cluster mass function from the matter power spectrum is performed with the formulas given by Tinker et al. (2008). Specifically, we have used their Eqs. (2) and (3) with the values $A=0.186$, $a=1.47, b=2.57$, and $c=1.19$ for the redshift zero mass function and applied their Eqs. (5) to (7) for the redshift evolution. To derive the predicted X-ray luminosity function from the theoretically calculated cluster mass function, we used empirical scaling relations of X-ray luminosity and mass (Böhringer et al. 2014, Eq. (10)) in accordance with the observations of Reiprich \& Böhringer (2002), Pratt et al. (2009) and Vikhlinin et al. (2009), within their confidence limits.

\footnotetext{
${ }^{1} r_{500}$ is the radius where the average mass density inside reaches a value of 500 times the critical density of the Universe at the epoch of observation.

2 While most of the X-ray observational data are limited to the region inside $r_{500}$, we use $M_{200}$ as cluster mass parameter for better comparison with the literature. The conversion from $M_{500}$ to $M_{200}$ is performed by an extrapolation based on the well established NFW profile (Navarro et al. 1995).

3 CAMB is publicly available from http://www.camb.info/ CAMBsubmit.html
}

The predicted X-ray luminosity function depends most strongly on the two cosmological parameters, $\Omega_{\mathrm{m}}$ and $\sigma_{8}$. Thus these are the parameters to be best constrained with the galaxy cluster data. The constraining power of the data is mostly limited by systematics, that is, by our imprecise knowledge of the cluster scaling relation of X-ray luminosity and mass (e.g. Böhringer et al. 2012). To derive realistic constraints on $\Omega_{\mathrm{m}}$ and $\sigma_{8}$ we therefore marginalise over the uncertainties in the two most influential and important parameters: we allow for a 7\% uncertainty in the slope of the scaling relation and an uncertainty of $14 \%$ in its normalisation (equivalent to the mass calibration) as $1 \sigma$ constraints of these parameters. We also include a scatter of the scaling relation of $30 \%$ and in addition a mass bias of 10\% (Böhringer et al. 2014). The latter accounts for the fact that the cluster masses in our empirical scaling relations, which have been determined from X-ray observations assuming hydrostatic equilibrium of the intracluster medium, are on average biased low. For more details on the marginalisation see Böhringer et al. (2014). The result of the likelihood analysis including this marginalisation is shown in Fig. $1^{4}$. It implies the following results, $\Omega_{\mathrm{m}}=0.285 \pm 0.04$ and $\sigma_{8}=0.776 \pm 0.07$.

\section{Method}

In the above analysis the mass function appears as an intermediate product. It is calculated for a given cosmological model, and is subsequently used to predict the X-ray luminosity distribution of the clusters which is fitted to the observational data. The nominal mass function is that which corresponds to the cosmological model fitting best to the observational data. To determine the uncertainty of the mass function we use the marginalisation approach described above. We sample the distribution of mass functions corresponding to the parameter distribution shown in Fig. 1. For each pair of cosmological parameters there is also a best fitting set of scaling relation parameters ${ }^{5}$. To determine the uncertainty of the mass function consistent with the cosmological model framework, we sample 100 parameter sets from statistical distribution shown in Fig. 1, and calculate 100 different mass functions accordingly. For this set of functions we determine the $16 \%$ and $84 \%$ percentiles as $1 \sigma$ limits.

We consider cosmologies without massive neutrinos and a model with a sum of the neutrino masses of $\sum m_{v}=0.4 \mathrm{eV}$. We also study the influence of the variation of the Hubble parameter in the range from $H_{0}=67$ to $73 \mathrm{~km} \mathrm{~s}^{-1} \mathrm{Mpc}^{-1}$.

\section{Results}

Figure 2 shows the results for the mass function implied by the best fitting cosmological model, the $1 \sigma$ uncertainties from the marginalisation and the uncertainties from allowing an additional flat prior on $H_{0}$ in the range between 67 and

\footnotetext{
4 The uncertainty in the theoretical modelling of the mass function has not been considered in the above error budget. It corresponds to a systematical uncertainty of a few percent, typically not more than $5 \%$ for $\Omega_{\mathrm{m}}$ and $\sigma_{8}$.

5 Including the variation of the scaling relation parameters is necessary for the following reason. For a fixed set of scaling parameters the error ellipse for $\Omega_{\mathrm{m}}$ and $\sigma_{8}$ is much smaller than shown in Fig. 1 (see Fig. 9 in Böhringer et al. 2014). For the full parameter range allowed by the constraint limits in Fig. 1, good fits to the mass function are only provided for all values of $\Omega_{\mathrm{m}}$ and $\sigma_{8}$ if the scaling relation parameters are also optimised. Therefore the mass function is only consistent with the data for certain combinations of cosmological and scaling relation parameters.
} 


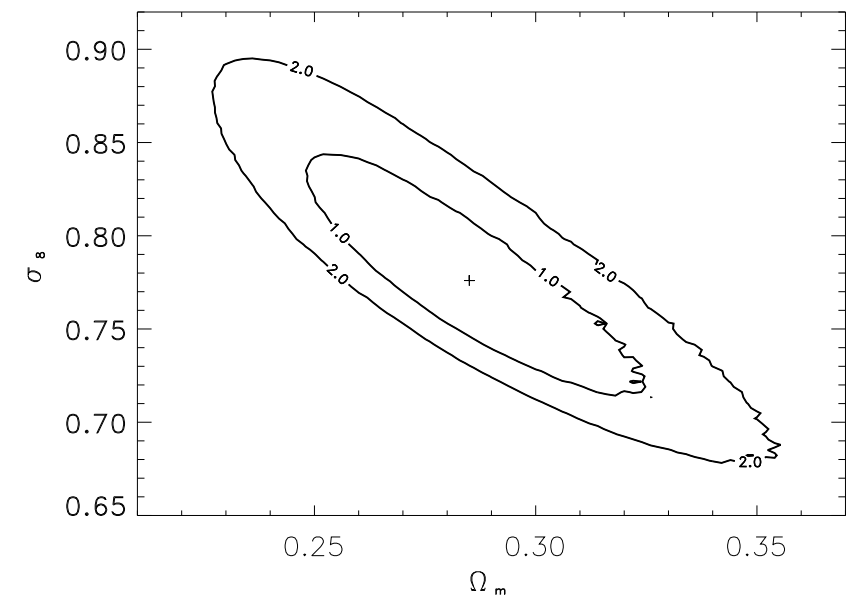

Fig. 1. Constraints on the cosmological parameters $\Omega_{\mathrm{m}}$ and $\sigma_{8}$ from the REFLEX galaxy cluster survey obtained by marginalising over the uncertainties in the cluster scaling relation for $\mathrm{X}$-ray luminosity and mass (from Böhringer et al. 2014). The cross shows the best fitting parameters and the ellipses indicate 1 and $2 \sigma$ uncertainties.

$73 \mathrm{~km} \mathrm{~s}^{-1} \mathrm{Mpc}^{-1}$. The cluster masses were calculated for a fiducial radius of $r_{200}$, the radius inside which the mean matter density is 200 times the critical density at the cluster redshift. We note that the mass function shown is what is implied for a redshift of zero, while the mass function used for the fit to the REFLEX cluster data is for a redshift of $z=0.102$. The cluster mass function is tightly constrained with an uncertainty less than $10 \%$ in the mass range $3 \times 10^{12}$ to $5 \times 10^{14} M_{\odot}$. The function is less well constrained at the high-mass end. The uncertainties are displayed in more detail in the lower part of the plot, which shows the ratio of the upper and lower limits to the best fitting function. We note that the variation of the Hubble constant in the above quoted range changes the results by an amount smaller than the uncertainties.

We compare these results of the mass function to data points of the REFLEX sample in the figure. These data have been obtained by converting the X-ray luminosity function from (Böhringer et al. 2014) to a mass function by use of the $L_{\mathrm{X}}-M$ scaling relation. This is over-plotted in Fig. 2 with data points for the binned mass function of the REFLEX sample with 20 clusters per bin, except for the bin at the lowest masses, which has only three clusters. Since the data correspond to a median redshift of $z=0.102$ we also plotted in Fig. 2 the mass function predicted for this redshift as a dashed line. This line fits the observational data very well. One can observe a dip of two to three data points at the low-mass end, which deviates from the line. This is mostly an effect of cosmic variance, caused by the matter underdensity in the nearby Universe in the southern sky, detected by our REFLEX II survey (Böhringer et al. 2015). Thus we conclude that the two derivations of the galaxy cluster mass function are consistent. We also note, that our new approach, including the consistency with structure formation theory, provides tighter constraints.

The results shown have been derived for a negligible mass of all neutrino families. Allowing for a minimum mass of neutrinos given by the constraints from particle physics of $\sum m_{v}=0.06 \mathrm{eV}$ has a hardly noticible effect on the results. To better demonstrate the effect of a neutrino mass we have taken a larger value of $\sum m_{v}=0.4 \mathrm{eV}$ (motivated also by the upper limit of our own cosmological constraints obtained with the REFLEX II survey in Böhringer \& Chon 2015). We find that the change is smaller than

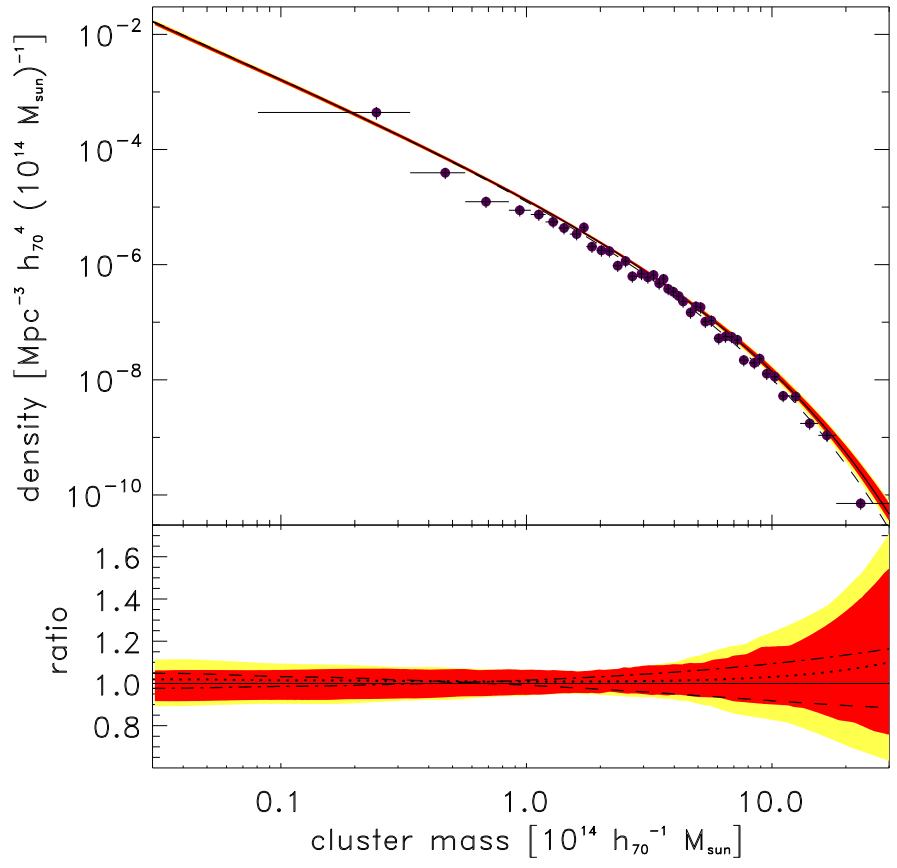

Fig. 2. Upper panel: mass function for the cosmological model best fitting the REFLEX Survey data (thick black line). The dark shaded region shows the uncertainties while marginalising over the $1 \sigma$ limits of the most important cosmological and scaling relation parameters (see text). The lightly shaded region shows in addition the variation with the Hubble constant in the range 67 to $73 \mathrm{~km} \mathrm{~s}^{-1} \mathrm{Mpc}^{-1}$. The data points give the mass function derived from the $\mathrm{X}$-ray luminosity function of the REFLEX cluster survey. The dashed line gives the best fitting mass function for a redshift of $z=0.102$ for comparison with the REFLEX data. Lower panel: ratio of the uncertainty limits to the best fitting function, where the light and dark shaded regions have the same meaning as above. The dashed and dashed-dotted lines show the ratio for the best fitting functions for a Hubble constant of 67 and $73 \mathrm{~km} \mathrm{~s}^{-1} \mathrm{Mpc}^{-1}$. The dotted line shows the best fitting function allowing the sum of the mass of the neutrinos to be $0.4 \mathrm{eV}$.

the uncertainties (as long as no further external observational data are included). This is shown in the lower panel of Fig. 2.

The tight constraints of the cluster mass function rely, however, on the precision of the mass function model by Tinker et al. (2008) used in our analysis. Therefore it is useful to explore how the function changes with the use of other published mass function models. In Fig. 3 we provide the results for the mass function based on models proposed by Watson et al. (2013) and Despali et al. (2016). The constraints shown for the use of Watson's algorithm are based on the model mass function for AHF halos defined as overdensities ${ }^{6}$. The results show reasonable agreement, with variations of up to $20 \%$ at the outer extremes of the displayed mass range. The function of Watson et al. predicts a smaller number of less massive clusters, while the model of Despali et al. predicts a slightly higher number of clusters than Tinker et al. (2008) over the whole mass range.

6 Watson et al. (2013) provide three different mass function models: one based on a friends-of-friends (FoF) halo finder, the AHF model defined by an overdensity threshold, and the CMPSO algorithm also based on spherical overdensity. Using the different models results in very similar mass function constraints with differences barely larger than the uncertainties. The largest difference is on the large mass end above $10^{15} M_{\odot}$, where the FoF method predicts more clusters than Tinker et al. (2008) and the spherical overdensity methods stay on average below. 


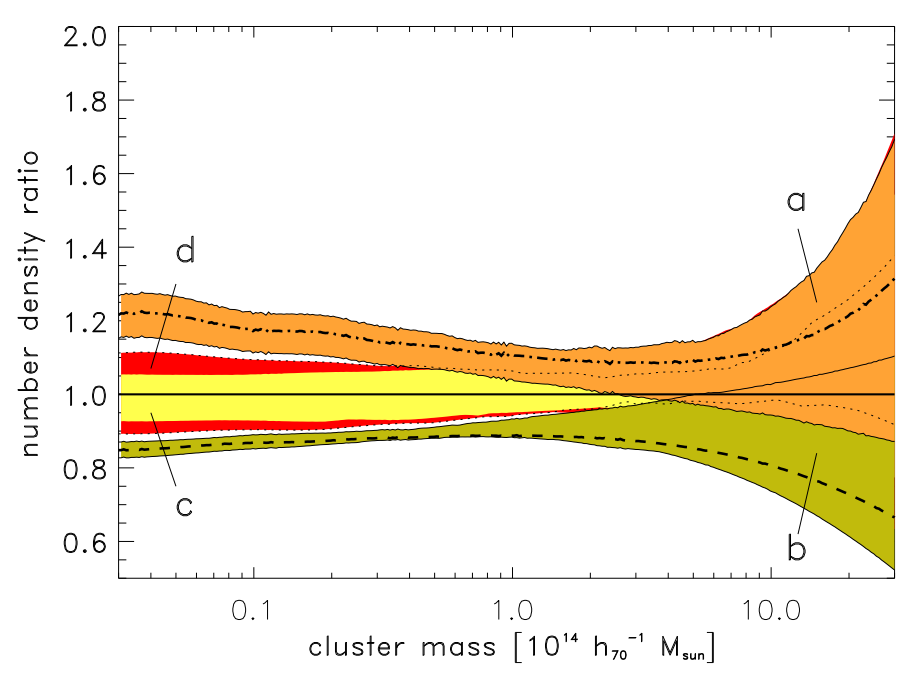

Fig. 3. Galaxy cluster mass function for the cosmological model best fitting the REFLEX Cluster Survey data based on different cluster mass function models. The mass functions are normalised to the results based on the Tinker mass function (solid line). The dashed line shows the results for the mass function model by Watson et al. (2013) with the uncertainty range labeled by (b), and the dashed-dotted line the results using the model by Despali et al. (2016) with uncertainties labeled by (a). The yellow-shaded region labeled (c) shows the uncertainties after marginalisation for the mass function based on Tinker et al. and the red region labeled (d) indicates the additional uncertainties from the marginalisation over the uncertainty in the Hubble parameter.

We note that, as a consequence of the difference in the best fitting cosmological parameters between Planck cosmic microwave background and clusters, the derived mass function is significantly lower than what is predicted by using cosmological parameters in accordance with the published results from the Planck mission (Planck Collaboration XX 2014, Planck Collaboration XXIV 2016). At the high-mass end, the cluster abundance is about a factor of two higher for Planck cosmology than for the mass function derived here. This has been illustrated in our earlier publications (Böhringer et al. 2014; Böhringer \& Chon 2015, 2016 - see in particular Fig. 11 of Böhringer et al. 2014) and reasons for this discrepancy have been discussed.

An accurate empirical mass function of galaxy clusters was also derived by Vikhlinin et al. (2009) from detailed Chandra observations of a flux limited sample of 49 nearby galaxy clusters. The total mass of these clusters has been derived, on one hand from good measurements of the total gas mass in the clusters and a calibration of the gas to total mass ratio from simulations, and on the other hand from observations of the total thermal energy in the clusters (gas mass multiplied by temperature) and the simulation calibrated scaling relation of this parameter with the total mass (Vikhlinin et al. 2009). Since the clusters of the Vikhlinin sample cover a redshift range from $z=0$ to 0.25 , we compare the mass function data points in Fig. 4 to the observationally constrained model predicted mass function for $z=0, z=0.1$, and $z=0.25$. As the publication by Vikhlinin et al. is based on a Hubble constant different from the one used here, we rescaled their data accordingly. We see that, apart from five data points at high mass, the model mass functions bracket the data very well. We actually observe that at low mass the data points are close to the $z=0$ function and at high mass they approach the $z=0.25$ function as expected for a flux limited sample, with low-mass objects very nearby and high-mass objects only sampled by the larger volume including higher reshifts.

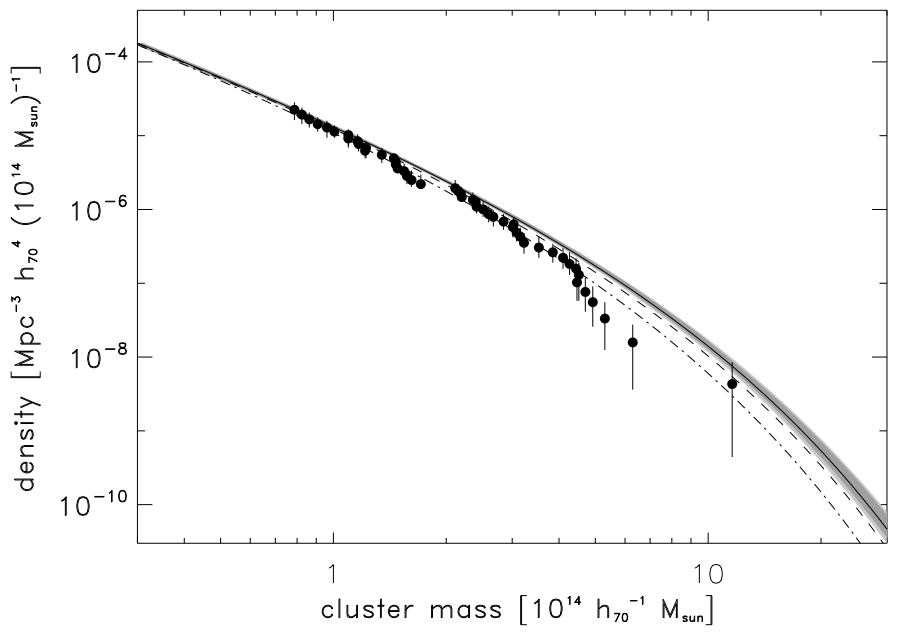

Fig. 4. Comparison of the observationally constrained mass function for redshifts of $z=0, z=0.1$, and $z=0.25$ with observational results by Vikhlinin et al. (2009) for a flux-limited sample of 49 clusters in the redshift range $z=0$ to 0.25 .

In Fig. 5 we derive the cumulative mass function, the cluster density for all clusters above a lower mass limit. This function was previously determined for example by Bahcall \& Cen (1993). We compare their results to our constraints in the Figure, where we have indicated the different cluster samples of these authors coming from a complete sample of Abell clusters (Abell 1958), from optical data for the EDCC clusters (Lumsden et al. 1992; Nichol et al. 1992), and X-ray clusters from Henry \& Arnaud (1991) using different symbols. Although the cluster number density is slightly overestimated from these data in the middle of the mass range, these early results are in remarkable agreement with our derivation.

While the mass function for REFLEX has been constructed for a mass of $M_{200}$, the masses by Bahcall \& Cen were originally determined for a radius of $1.5 h_{100}^{-1} \mathrm{Mpc}$ and different cosmological parameters. To make the comparison, we have converted their masses to our fiducial cosmological model and we have also translated the mass results to a radius of $r_{200}$ by means of the NFW mass profile (Navarro et al. 1995) with a concentration parameter of 5 .

\section{Application}

\subsection{Fits to the cumulative mass function}

For an easy application of our results to cosmological modelling, we provide an analytic fit to the derived cumulative mass function at $z=0$. A Schechter function provides an unsatisfactory fit to the data; the function decreases too fast at high mass. We found the following function with four free parameters to provide a better fit:

$n(>M)=\alpha\left(\frac{M}{2 \times 10^{14} h_{70}^{-1} M_{\odot}}\right)^{-\beta} \exp \left(-\frac{M^{\delta}}{\gamma}\right)$.

Figure 5 shows the deviation of this fit from the observationally derived function. Within a mass range of $3 \times 10^{12}-10^{15} h_{70}^{-1} M_{\odot}$ the fit reproduces the mass function with an error of less than $5 \%$. In the figure we also show the uncertainty of the mass function obtained by the marginalisation over the important cosmological and scaling parameters not including the variations with 
H. Böhringer et al.: REFLEX II: the galaxy cluster mass function

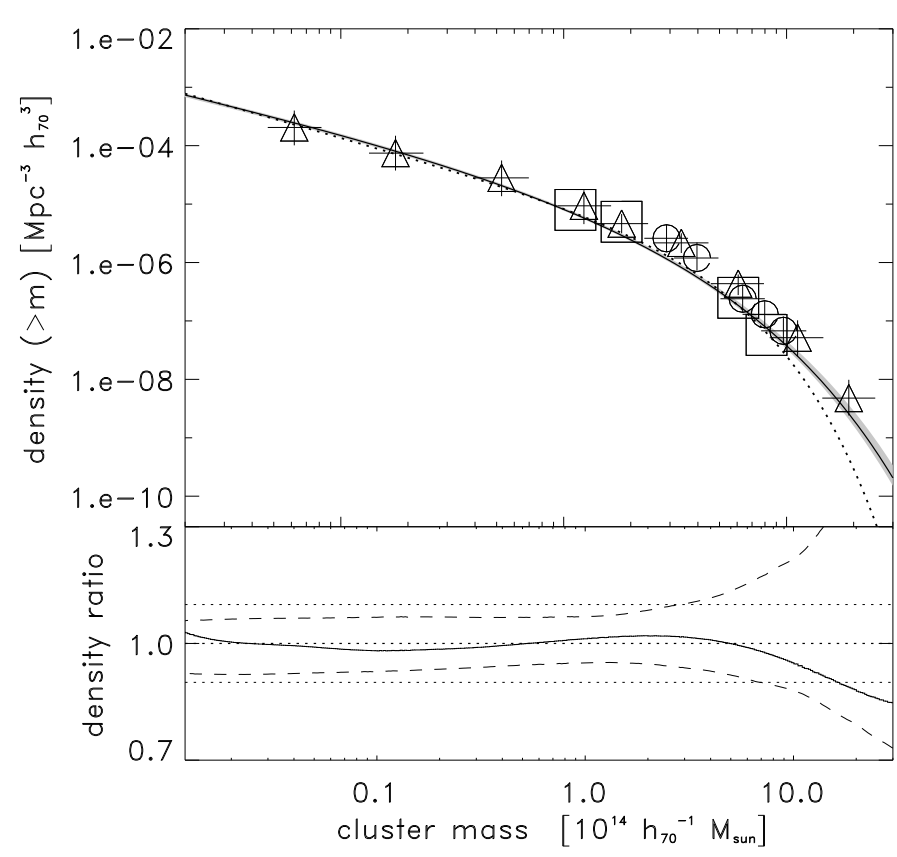

Fig. 5. Upper panel: cumulative cluster mass function for the cosmological model best fitting the REFLEX Survey data and its uncertainties (grey region). The present data are compared to a previous determination of the cumulative mass function of clusters by Bahcall \& Cen (1993), shown by data points. The different data points relate to different cluster samples used by Bahcall \& Cen: Abell clusters (triangles with error bars), EDCC clusters (squares), and X-ray clusters (circles with error bars). The dotted curve shows the best fitting Schechter function, which falls short of describing the high end of the mass function. Bottom panel: ratio of the fitted function to the true cumulative mass function. We also show the uncertainties of the mass function from the marginalisation not including the varying Hubble parameter (dashed lines). The dotted lines indicate $10 \%$ deviations.

Table 1. Fit parameters for the approximations to the cumulative mass function.

\begin{tabular}{lrrrr}
\hline \hline Function & $\alpha^{1}$ & $\beta$ & $\gamma^{2}$ & $\delta$ \\
\hline Best fit & 1.237 & 0.907 & 0.961 & 0.625 \\
Fit to lower limit & 1.205 & 0.895 & 0.941 & 0.627 \\
Fit to upper limit & 1.231 & 0.922 & 1.026 & 0.629 \\
Fit lower limit incl. $H_{0}$ var. & 1.252 & 0.980 & 0.907 & 0.625 \\
Fit upper limit incl. $H_{0}$ var. & 1.163 & 0.942 & 1.083 & 0.636 \\
Fit incl. massive neutrinos & 1.367 & 0.904 & 0.895 & 0.608 \\
Best fit, 3 free parameters & 0.821 & 1.000 & 1.372 & 0.710 \\
\hline
\end{tabular}

Notes. (1) $\alpha$ in units of $10^{-5} \mathrm{Mpc}^{-3} h_{70}^{3} 10^{14} M_{\odot}$; (2) $\gamma$ in units of $\left(10^{14} M_{\odot}\right)^{\delta}$.

$H_{0}$. The deviations of the fitted functions are smaller than the uncertainties.

In Table 1 we provide the parameters for the best fit, for the lower, and upper $1 \sigma$ limit without and with marginalisation over the Hubble parameter. We also give the fit to the cumulative mass function obtained for a cosmology with a total neutrino mass of $\sum_{i} m_{v_{i}}=0.4 \mathrm{eV}$. In addition we provide a fit for the best fitting mass function with the parameter $\beta$ in Eq. (1) fixed to a value of 1 . This three-parameter fit is also an acceptable representation with less than $10 \%$ deviation in the relevant mass range.

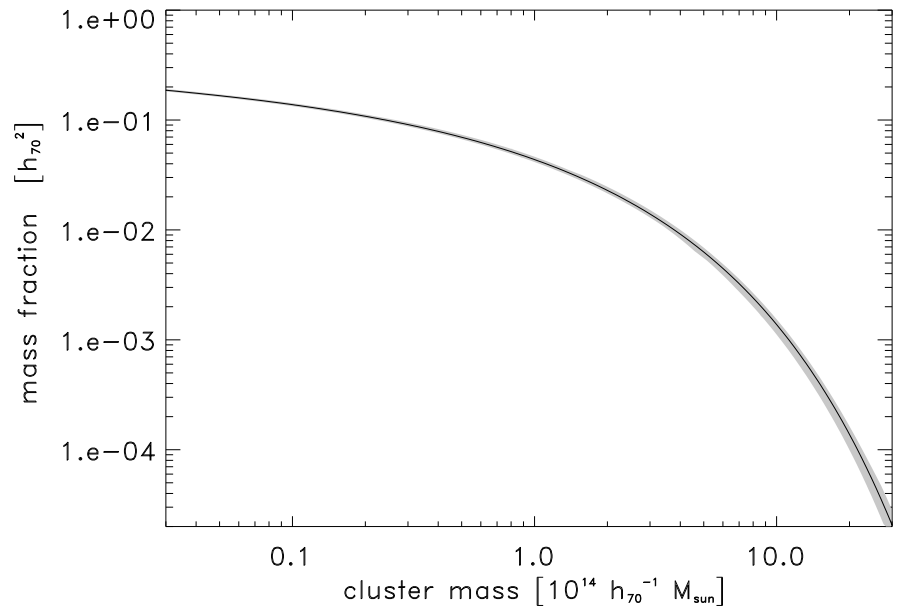

Fig. 6. Mass fraction bound in groups and clusters of galaxies inside $r_{200}$ at a redshift of zero.

\subsection{Cosmic mass fraction in cluster and groups}

The cumulative mass function allows us to calculate the fraction of matter in the Universe contained in clusters and groups above a certain mass limit inside a radius of $r_{200}$. This is shown in Fig. 6, where the mass fraction in groups and clusters has been normalised by the total amount of matter in the Universe for $H_{0}=70 \mathrm{~km} \mathrm{~s}^{-1} \mathrm{Mpc}^{-1}$ and the relevant value of $\Omega_{\mathrm{m}}$ which is 0.285 for the best-fitting model. At present, about $14 \pm 1 \%$ of the matter in the Universe is contained in groups and clusters with a mass above $10^{13} h_{70}^{-1} M_{\odot}$, and $4.4 \pm 0.4 \%$ is locked up in clusters with a mass above $10^{14} h_{70}^{-1} M_{\odot}$ inside a radius of $r_{200}$. If we extend the calculations to higher redshifts, we find only of the order of $1 \%$ of the matter is bound in groups and clusters with $M_{200}>10^{13} h_{70}^{-1} M_{\odot}$ at a redshift of 2 , illustrating the recent rapid evolution of the cluster population.

\subsection{Evolution of the mass function with redshift}

We can use our constraints on the evolution of structure formation to derive further constraints on the cluster population as a function of redshift. Figure 7 shows the cumulative number of clusters that can be observed out to a maximum redshift as a function of the lower cluster mass limit. The total number of groups and clusters out to a redshift of 2 is for example predicted to be about $36 \pm 2.5$ million with a mass above $M_{200}>10^{13} h_{70}^{-1} M_{\odot}$ and for clusters with a mass above $M_{200}>10^{14} h_{70}^{-1} M_{\odot}$ about $560000 \pm 60000$.

We also note in Fig. 7 that the increase in number counts at higher redshifts only involves the lighter systems. Thus for more massive systems, there is practically a limiting redshift, beyond which we cannot expect to find further objects of this size. This is further detailed in Fig. 8. We show here the increase in number counts for systems with $M_{200}>2 \times 10^{15} M_{\odot},>3 \times$ $10^{14} h_{70}^{-1} M_{\odot}$, and $>10^{14} h_{70}^{-1} M_{\odot}$. We thus expect to find only about $9-10$ clusters with a mass above $2 \times 10^{15} h_{70}^{-1} M_{\odot}$ and they should all reside at redshifts lower than 0.75 . In the whole sky we should find about 390 clusters with $M>1 \times 10^{15} h_{70}^{-1} M_{\odot}$, with the most distant four clusters found in the redshift interval $z=1.0-1.2$.

We can compare this to the actual numbers found in our survey. For REFLEX II and NORAS II together (which we now call the CLASSIX survey), which cover about two thirds of the 


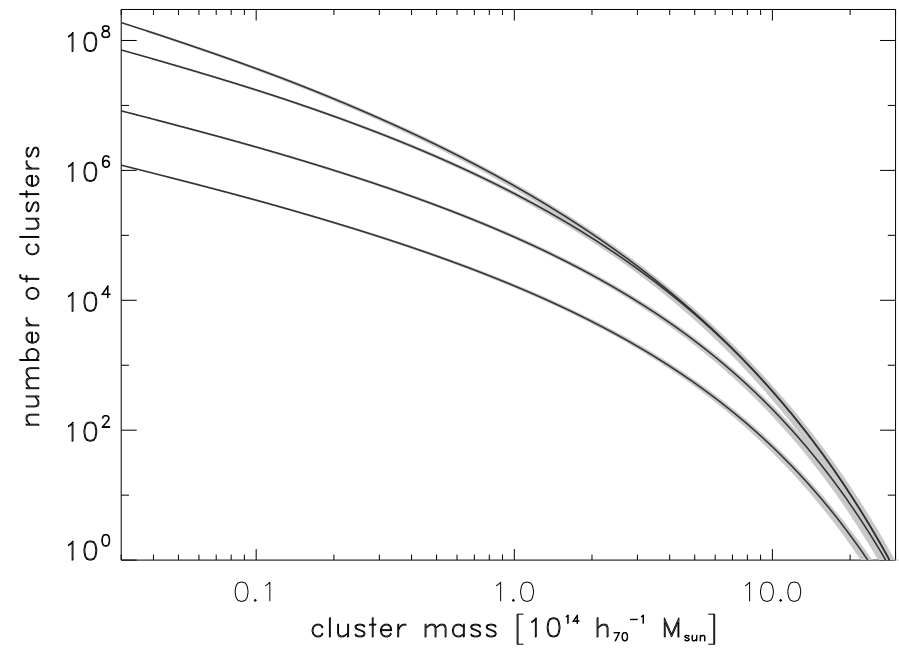

Fig. 7. Cumulative number counts of clusters in the full sky as a function of mass for different limiting redshifts. The curves show the cut-off redshifts of $z=0.2,0.4,1.0$, and 2.0 , respectively.

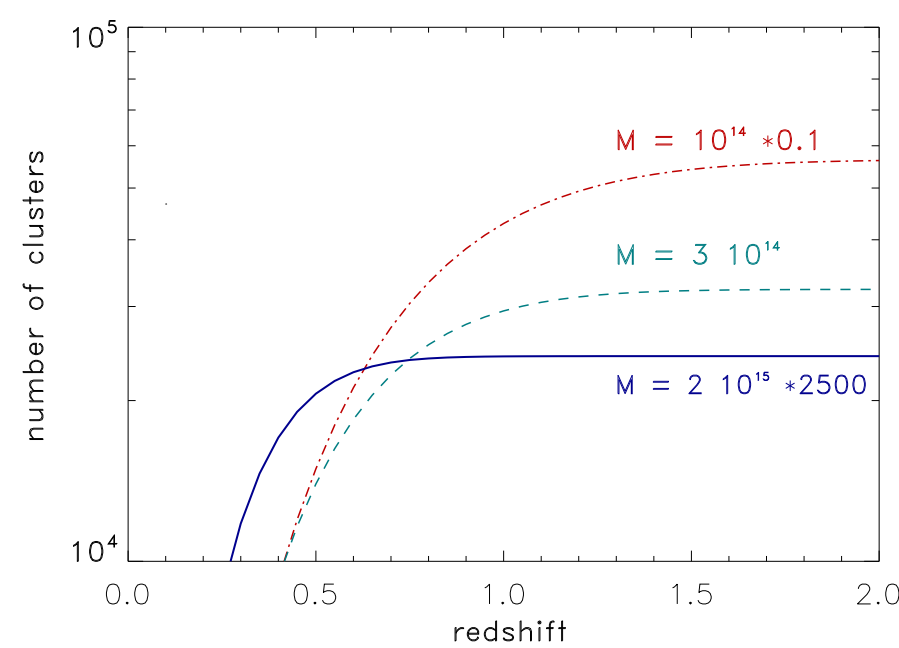

Fig. 8. Reshift evolution of the cumulative number counts of clusters for different minimum mass. The lower mass cut-off for the curves are from top to bottom $10^{14} h_{70}^{-1}, 3 \times 10^{14} h_{70}^{-1}$, and $2 \times 10^{15} h_{70}^{-1} M_{\odot}$.

sky (8.25 ster), we find 16 clusters with a scaling relation determined mass $>2 \times 10^{15} h_{70}^{-1} M_{\odot}$. This number is larger than the expected value quoted above because the scaling relations have a considerable scatter, which strongly affects the number counts at the high mass end, where the mass function is particularly steep. If we include a $30 \%$ scatter in the X-ray luminosity mass relation, we predict to find 19 high-mass clusters, of which 13 should lay in the CLASSIX survey ${ }^{7}$ region. For clusters with $M_{200}>10^{15} h_{70}^{-1} M_{\odot}$ we predict about 200 such objects for $z<0.4$. Including a $30 \%$ scatter and accounting for the sky coverage of CLASSIX we predict 183 clusters and observe 161 . Thus, taking into account the scatter in the scaling relations with the same amount as used for the cosmological modelling, we get a good agreement with the predictions and the observations.

\footnotetext{
7 Cosmic Large-Scale Structure in X-rays Cluster Survey (Böhringer
} et al. 2016).

\section{Summary and conclusion}

A major goal of this paper is to provide the mass function of galaxy clusters as a census of the galaxy cluster population in the low-redshift Universe. We have shown that combining our catalogue of X-ray luminous clusters from the REFLEX survey with structure-formation theory, we obtain a mass function with small uncertainties not larger than $10 \%$ in the mass range $3 \times$ $10^{12} h_{70}^{-1}$ to $5 \times 10^{14} h_{70}^{-1} M_{\odot}$. We also provided approximations by parameterised functions, which capture the results well within their uncertainties.

The largest uncertainties included in these results come from the limited precision of the mass-observable scaling relations of galaxy clusters, which is also the major limit factor in modelling galaxy cluster cosmology. Large efforts are currently being made to improve this situation and we hope that this will enable us to derive a more precise cluster mass function and its evolution in the future. We also find that there is a significant uncertainty on the theoretical side with the mass function model. If we want to improve the results well below a $10 \%$ accuracy, both the modelling of the cluster mass function and the halo definition from simulations must be advanced.

We have used our results for some exploration of the evolution of the cluster population with redshift, and we show how the most massive clusters are confined to the low-redshift range. Ongoing and future surveys over a larger redshift range will further tighten constraints on the mass function. But here the situation is even more severe due to the uncertainties in scaling relations, since the observational data on the relations are more sparse and less precise at higher redshift. Therefore, future surveys will need to provide not only better samples but also a means to improve the mass-observable scaling relations. In this respect the Euclid mission with its capability of providing lensing masses for a large number of groups and clusters (e.g. Sartoris et al. 2016) offers especially interesting prospects.

Acknowledgements. H.B. and G.C. acknowledge support from the DFG Transregio Program TR33 and the Munich Excellence Cluster "Structure and Evolution of the Universe". G.C. acknowledges support by the DLR under grant No. 50 OR 1405. M.F. thanks Yasuo Tanaka at the Max-Planck-Institut fuer Extraterestische Physik and the Alexander von Humboldt Stiftung for their support during his stay in Garching. We would like to thank the referee for useful comments which helped to make some points clearer.

\section{References}

Abell, G. O. 1958, ApJS, 3, 211

Bahcall, N., \& Cen, R. 1993, ApJ, 407, L49

Bardeen, J. M., Bond, J. R., Kaiser, N., \& Szalay, A. 1986, ApJ, 304, 15

Berlind, A. A., \& Weinberg, D. H. 2002, ApJ, 575, 587

Böhringer, H., \& Chon, G. 2015, A\&A, 574, L8

Böhringer, H., \& Chon, G. 2016, Mod. Phys. Lett. A, 31, 1640008

Böhringer, H., Voges, W., Huchra, J. P., et al. 2000, ApJS, 129, 435

Böhringer, H., Schuecker, P., Guzzo, L., et al. 2001, A\&A, 369, 826

Böhringer, H., Schuecker, P., Guzzo, L., et al. 2004, A\&A, 425, 367

Böhringer, H., Schuecker, P., Pratt, G. W., et al. 2007, A\&A, 469, 363

Böhringer, H., Dolag, K., \& Chon, G. 2012, A\&A, 539, A120

Böhringer, H., Chon, G., Collins, C. A., et al. 2013, A\&A, 555, A30

Böhringer, H., Chon, G., Collins, C. A., et al. 2014, A\&A, 570, A31

Böhringer, H., Chon, G., Bristow, M., et al. 2015, A\&A, 574, A26

Böhringer, H., Chon, G., \& Kronberg, P. P. 2016, A\&A, 596, A22

Böhringer, H., Chon, G., Retzlaff, J., et al. 2017, AJ, 153, 220

Bond, J. R., Cole, S., Efstathiou, G., \& Kaiser, N. 1991, ApJ, 379, 440

Despali, G., Giocoli, C., Angulo, R. E., et al. 2016, MNRAS, 456, 2486

Henry, J. P. 2004, ApJ, 609, 603

Henry, J. P., \& Arnaud, K. A. 1991, ApJ, 372, 410 
H. Böhringer et al.: REFLEX II: the galaxy cluster mass function

Jenkins, A., Frenk, C. S., White, S. D. M., et al. 2001, MNRAS, 321, 372 Lesgourges, J., \& Pastor, S. 2006, Phys. Rep., 429, 307

Lewis, A., Challinor, A., \& Lasenby, A. 2000, ApJ, 538, L473

Lumsden, S. L., Nichol, R. C., Collins, C. A., et al. 1992, MNRAS, 258, 1

Mantz, A., Allen, S. W., Rapetti, D., et al. 2010, MNRAS, 406, 1759

Mehrtens, N., Romer, A. K., Nichol, R. C., et al. 2016, MNRAS, 463, 1929

Navarro, J. F., Frenk, C. S., \& White, S. D. M. 1995, MNRAS, 275, 720

Nichol, R. C., Collins, C. A., Guzzo, L., et al. 1992, MNRAS, 255, 21

Peacock, J. A., \& Smith, R. E. 2000, MNRAS, 318, 1144

Perrenod, S. C. 1980, ApJ, 236, 373

Planck Collaboration XX. 2014, A\&A, 571, A20

Planck Collaboration XXIV. 2016, A\&A, 594, A24
Pratt, G. W., Croston, J. H., Arnaud, M., et al. 2009, A\&A, 498, 361 Press, W. H., \& Schechter, P. 1974, ApJ, 187, 425

Reiprich, T. H., \& Böhringer, H. 2002, ApJ, 567, 716

Rozo, E., Wechsler, R. H., Rykoff, E. S., et al. 2010, ApJ, 708, 645

Sartoris, B., Biviano, A., Fedeli, C., et al. 2016, MNRAS, 459, 1764

Schechter, P. L. 1976, ApJ, 203, 297

Tinker, J., Kravtsov, A. V., Klypin, A., et al. 2008, ApJ, 688, 709

Trümper, J. 1993, Science, 260, 1769

Vikhlinin, A., Kravtsov, A. V., Burenin, R. A., et al. 2009, ApJ, 692, 1060

Voges, W., Aschenbach, B., Boller, T., et al. 1999, A\&A, 349, 389

Warren, M. S., Abazajian, K., Holz, D. E., et al. 2006, ApJ, 646, 881

Watson, W. A., Iliev, I. T., D’Aloisio, A., et al. 2013, MNRAS, 433, 1230 